\title{
TNT biotransformation potential of the clinical isolate of Salmonella typhimurium - potential ecological implications
}

\begin{abstract}
Out of fifty-six isolates screened three bacterial strains enriched with TNT either as sole source of nitrogen (for Salmonella typhimurium, Klebsiella pneumoniae) or along with co-substrate (for Acinetobacter baumannii), have been carried out nitro group reduction under aerobic conditions. During studies, S. typhimurium found to have high potential $\left(100 \%\right.$ of $\left.50 \mathrm{mg} \mathrm{l}^{-1}\right)$ in removal of TNT, than $K$. pneumoniae $\left(70 \%\right.$ of $\left.20 \mathrm{mg} \mathrm{l}^{-1}\right)$ and $A$. baumannii (52\% of $40 \mathrm{mg} \mathrm{l}^{-1}$, in presence of co-substrate). Therefore studies were focused on $S$. typhimurium, which had shown good growth, and protein contents, with disappearance of TNT, and concomitantly release of nitrite over the period of time. Removal of TNT was analyzed by HPLC, and nitrite liberation was consistently found coincided with TNT disappearance from the medium. As compared to earlier reports, $100 \%$ disappearance of TNT within $30 \mathrm{~h}$ by $S$. typhimurium is encouraging, and may indicate its potential in bioremediation of TNT. This is the first report on $S$. typhimurium, Klebsiella pneumoniae and Acinetobacter baumannii for transformation of TNT with nitrite release into the medium.
\end{abstract}

Key Words: Acinetobacter baumannii, Bioremediation, Klebsiella pneumoniae, Salmonella typhimurium, TNT

\section{INTRODUCTION}

TNT (2, 4, 6-trinitrotoluene) is commonly used explosive, and its contamination is of a great concern because of the toxicity and mutagenicity. ${ }^{[1]}$ TNT concentrations at manufacturing, processing and disposal sites can be as high as $100 \mathrm{mg} / \mathrm{l}$ in water and $12000 \mathrm{mg} / \mathrm{kg}$ in soil. Numerous cases of munitions workers reportedly developed liver damage and anaemia owing to TNT exposure ${ }^{[2]}$ Bioremediation offers as attractive and economic way of removal of explosives from contaminated sites. ${ }^{[3]}$ Number of bacteria and fungi are able to utilize $\mathrm{TNT}^{[4]}$ as a nitrogen source by removing nitrogen under either aerobic ${ }^{[5]}$ or anaerobic conditions. ${ }^{[4,6]}$ Traditionally, most of the research on TNT bioremediation was focused on anaerobic bacteria and fungi, and only recently studies begun to appear wherein aerobic bacteria degrade TNT. Under many typical setting the rates of degradation are limited, mainly due to the accumulation of intermediate metabolites, and their toxic effects on biological systems. ${ }^{[2]}$

The investigators from various places have isolated several organisms, which degrade explosive aerobically. Most notable among them are Stenotrophomonas, ${ }^{[7]}$ Rhodococcus, ${ }^{[8,9]}$ Enterobacter ${ }_{,}^{[5]}$ Pseudomonas, ${ }^{[10]} \mathrm{My}$ cobacterium, $^{[11]}$ Bacillus, $^{[2]}$ Serratiad ${ }^{[12]}$ and Staphylococcus, ${ }^{[2]}$ Halasz et al. ${ }^{[13]}$ have reported detection of degradation of explosives by soil indigenous organisms.

A present study demonstrates the removal of TNT from culture media by locally isolated aerobic species viz. Salmonella typhimurium, Klebsiella pneumoniae, and Acinetobacter baumannii, which were successfully tolerated, sustained, removed, and grown in TNT enriched media. These organisms are not previously reported for his type of activity, and may prove to have potential to transform TNT. The study presented is focused $S$. typhimurium, which removed 100\% TNT.

\section{MATERIALS AND METHODS}

Chemicals, reagents, and media The stock solution of TNT (provided by Defence Research and Development Organization, Government of India) prepared in sterile de-ionized water, was filter $(0.22 \mu \mathrm{m}$, Satorius, Germany) sterilized and used as 100 $\mathrm{mg} / \mathrm{l}$. A range of TNT (1 through 100 $\mathrm{mg} / \mathrm{l}$ ) concentration was used to add into mineral medium as the sole source of nitrogen. The mineral me-
Geetanjali M. Litake, Suresh G. Joshi, Vikram S. Ghole Division of Biochemistry, Department of Chemistry, University of Pune, Pune - 411 007, India

For correspondence: Suresh G. Joshi, Department of Chemistry, University of Pune, Pune - 411 007, India. E-mail: surejoshi@yahoo.com

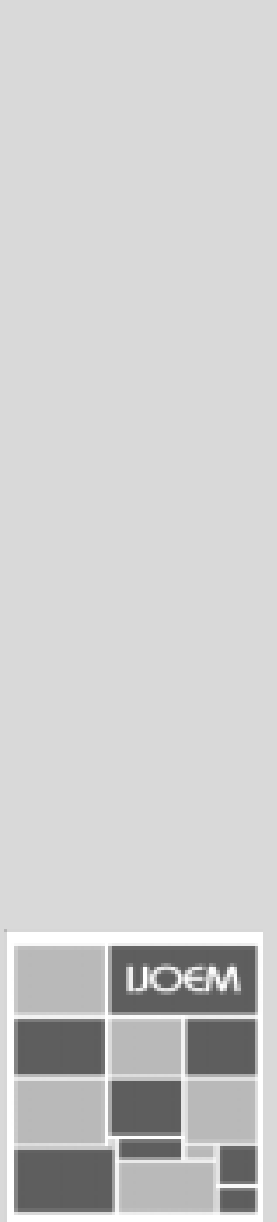


dium with some modification was used for evaluation of tolerance and degradation of TNT, the constituents of which were D-glucose (5mM), $\mathrm{MgSO}_{4}: 7 \mathrm{H}_{2} \mathrm{O}(0.02 \%), \mathrm{K}_{2} \mathrm{HPO}_{4}(0.7 \%), \mathrm{KH}_{2} \mathrm{PO}_{4}$ $(0.2 \%)$, sodium acetate $(0.1 \%)$, tri-sodium citrate $(0.05 \%)$, and $\left(\mathrm{NH}_{4}\right)_{2} \mathrm{SO}_{4}(0.1 \%)$. For biodegradation studies nitrogen deficient mineral medium was used wherein $\left(\mathrm{NH}_{4}\right)_{2} \mathrm{SO}_{4}$ was replaced with TNT. All chemicals were of analytical grade (Sigma Chemical Co., St. Louis, MO, USA) and solvents HPLC grade (Glaxo-Qualigen or Thomas Baker, India). Other culture media were obtained from either Difco (Detroit, MI, USA) or HiMedia (Bombay, India) Laboratories.

\section{Isolation of bacterial strains, growth conditions and measurement}

The random sample of soil and water were collected from the vicinity of ammunition plant, and its wastewater disposal stream meeting to river. In brief inoculum was prepared by suspending $1 \mathrm{~g}$ of soil or $1 \mathrm{ml}$ of wastewater (collected from the vicinity of munitions plant) in $9 \mathrm{ml}$ sterile distilled water, mixed thoroughly and allowed to settle down. The supernatant fluid was filtered through Whatman no. 1 filter and the filtrate was used as an inoculum. A loopful of the filtrate was streaked on nutrient agar (Difco) plates and incubated at an ambient temperature. The microbial colonies obtained were used to prepare secondary culture and identified according to standard methods desribed. ${ }^{[14,15]}$ A single isolated colony from purified secondary culture plate was inoculated into 30 $\mathrm{ml}$ of nutrient broth to grow over night. One $\mathrm{ml}$ of this over night grown culture was then inoculated into $100 \mathrm{ml}$ mineral medium containing TNT as nitrogen source in $500 \mathrm{ml}$ flat bottom conical flask and incubated either keeping cultures stationary or on rotary shaker (100 rpm) at ambient temperature. The cell density of liquid culture was determined spectrophotometrically as optical density at $600 \mathrm{~nm}$. The viable cell colonies were counted by serial dilutions plating on LB plates, whereas protein concentration estimated as described by French et $a l^{[5]}$ The incubation time for TNT experiments was up to $96 \mathrm{hr}$. The effect of $0.1 \%$ yeast extract (as growth enhancer) on initiation of growth of inoculated organism was also investigated. We isolated and screened 44 environmental, and 12 clinical isolates (laboratory strains originally collected for other clinical studies), for degradation activity of TNT. Abiotic cultures were performed for all the times as negative controls, and the cultures wherein DNT (Sigma) was limiting and sole source of nitrogen, as positive controls for the confirmation that these bacteria take aerobic path of transformation of TNT. Experiments were repeated minimum three times to reproduce the results, unless otherwise stated.

\section{HPLC monitoring for removal of TNT}

The disappearance of TNT was monitored, and quantified by testing the culture supernatants (obtained after centrifugation of culture at 6000 rpm for 15 min.) using High Performance Liquid Chromatography (HPLC) during cultivation at regular intervals. The HPLC analysis was performed with Perkin-Elmer system consisting of a biocompatible binary pump 250 and Lambda 3B UV/VIS spectrophotometer as detector. The Borwin version 1.21 (Jasco, Japan) software was

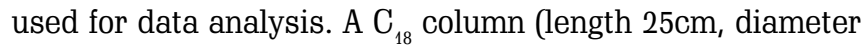
$4 \mathrm{~mm}$ (ID), and make VYDAC $\left(\mathrm{C}_{18}\right)$, USA) was used to analyze supernatants, using $20 \mathrm{ml}$ of sample injected for detection of TNT. An isocratic system was used for all the experiments. The mobile phase for TNT estimation was methanol: water (50:50 v/v), delivered at a flow rate of $1.0 \mathrm{ml} / \mathrm{min}$. The TNT was monitored at $230 \mathrm{~nm}$.

\section{Nitrite Assays}

A demineralization of TNT in the form of release of nitrites during growth of organism was assayed colorometrically as described previously ${ }^{[5]} \mathrm{In}$ brief, to $1 \mathrm{ml}$ sample aliquot, $0.4 \mathrm{ml}$ $\mathrm{N}$ - (1-naphthyl) ethylenediamine-sulfonilamide solution containing $0.3 \mathrm{M}$ sulfanilic acid and $0.5 \% \mathrm{~N}$ - (1-naphthyl) ethylenediamine-sulfanilamide dichloride were added. The reaction mixture was incubated for $20 \mathrm{~min}$ at room temperature and the OD at $543 \mathrm{~nm}$ was determined. Sodium nitrite was used as a standard.

\section{RESULTS}

\section{TNT as sole nitrogen source during growth of iso- lates}

The organisms were inoculated in mineral medium containing either $\left(\mathrm{NH}_{4}\right)_{2} \mathrm{SO}_{4}$ or TNT or DNT or no nitrogen source to observe their growth patterns and utilization of the nitrogen by aerobic pathway. The initial inoculi for all different organisms was about $1.6 \times 10^{6}$ cells/l. Growth was monitored spectrometrically and measured as optical density at $600 \mathrm{~nm}$ $\left(\mathrm{OD}_{600}\right)$. Parallel cultures of the same organisms were grown in known and easily available nitrogen source $\left[\left(\mathrm{NH}_{4}\right)_{2} \mathrm{SO}_{4}\right]$ or nitrogen deficient medium to compare the status of growth. Cultures grown in DNT were only for the purpose of monitoring its fate during incubation with bacteria under the similar conditions (that of TNT). In cultures containing variable initial concentrations of TNT, it is observed that TNT removal was associated with growth. The most promising growth was observed in two organisms, both of which were clinical isolates. The isolates of $S$. typhimurium and $K$. pneumoniae have removed respectively $100 \%$ (50 mg/l), and $70 \%$ (of $20 \mathrm{mg} / \mathrm{l}$ ) of TNT very efficiently during growth, without any growth enhancer or growth promoting substances. Very few aerobic organisms able to use TNT as a sole nitrogen source have been reported and much less frequently about mineralization of this compound. ${ }^{[4]}$ While testing the effect of $0.1 \%$ yeast extract (as co-substrate) on TNT biotransformation, we observed $A$. baumannii involved in significant transformation of TNT ( $52 \%$ of $40 \mathrm{mg} / \mathrm{l})$, which it failed to do so in the absence of this co-substrate. These organisms could utilized DNT for their growth during cultivation, indicating that these 
organisms take aerobic pathway (data not shown). None of these three species have earlier been reported to transform TNT. S. typhimurium has removed $100 \%$ of TNT ( $50 \mathrm{mg} / \mathrm{l}$, highest among all tested strains), and therefore analysis of the results pertaining to this organism have been presented. Although Acinetobacter johnsonii and Klebsiella sp. have been reported to biotransform TNT $(<10 \mathrm{mg} / \mathrm{l})$, our isolates are totally different species ( $A$. baumannii and $K$. pneumoniae) which removed TNT $>10 \mathrm{mg} / . .^{[16]}$

The growth and protein curves for $S$. typhimurium obtained during studies are shown in Figure 1a and 1b. Growth estimated by turbidity and proteins concentration was determined $i$ in the presence of either TNT or $\left(\mathrm{NH}_{4}\right)_{2} \mathrm{SO}_{4}$ was pro-
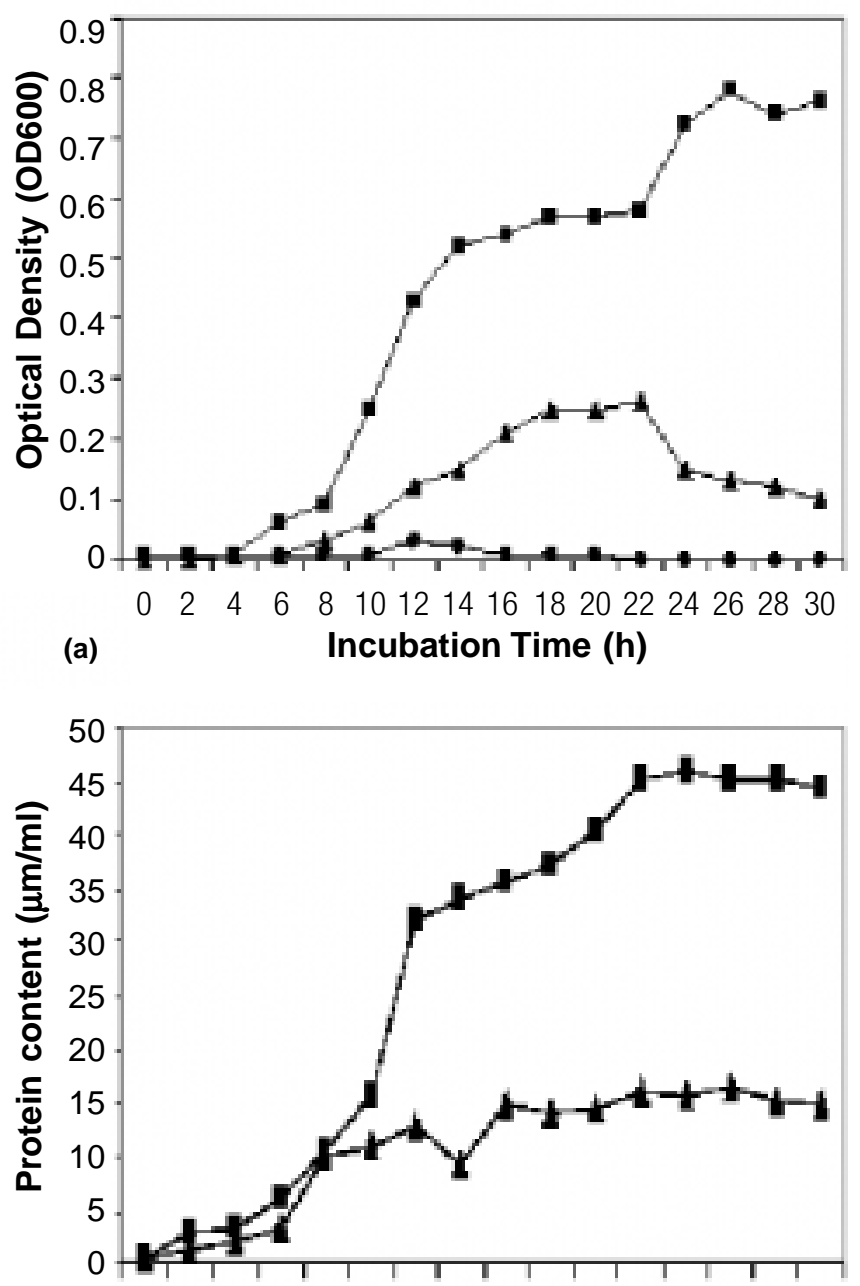

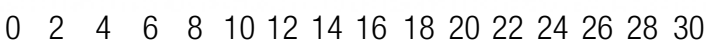
(b)

\section{Incubation Time (h)}

Figure 1: The representative graphs showing evidence of growth of $S$. typhimurium in TNT $\left(50 \mathrm{mg} \mathrm{l}^{-1}\right)$ enrich and minimal mineral medium. (a) growth measured as optical density at 600 $\mathrm{nm}$. (b) shows protein concentrations. The results shown are the mean of three independent experiments, and the error bar indicates standard error mean [ammonium sulphate (\%), TNT $(2 \%)$ or no nitrogen source $(\cdot)]$ portional to the amount of nitrogen source present in the growth medium. Viable cell count was higher in growth medium containing $\left(\mathrm{NH}_{4}\right)_{2} \mathrm{SO}_{4}$ than TNT (data not shown). To monitor the influence of TNT and its metabolites on cell viability, $S$. typhimurium from the liquid cultures of variable time points during incubation was plated on LB. The analysis exhibited good growth, and maintained cell viability in the presence of TNT up to $36 \mathrm{hr}$, indicating that TNT or its metabolites do not inhibit growth, and have no toxic effect on this strain up to the given time points. It also indicated that the initial inoculum was sufficient to initiate growth in the presence of TNT. In enriched medium, the isolate observed to tolerate TNT up to $60 \mathrm{mg} / \mathrm{l}$, and thereafter no tolerance, and rapid decreased in viable cell count, and inhibition of growth was observed. The optimal concentration of TNT was $50 \mathrm{mg} / \mathrm{l}$. In Figure 1a, the lag phase appeared to increase up to $8 \mathrm{~h}$, which corresponds with protein curve (Figure 1b). A change in color of medium was noted during cultivation. The medium started turning yellowish to slight orange around 4 $\mathrm{h}$, and continued darkening until $8 \mathrm{~h}$, around which the medium was pinkish red, which turned reddish brown when continued till $10 \mathrm{~h}$. The color of the medium after $12 \mathrm{~h}$ again turned yellow. Once the growth was initiated, the growth rate, protein contents, and terminal cell densities were proportional. The results were consistent through out, and the experiments were repeated thrice to check their reproducibility.

\section{Disappearance of TNT from culture medium}

Data from HPLC analysis showed that TNT was removed from the mineral medium during growth (Figure 2a, 2b). Two prominent peaks were observed. The first one (retention time, $\sim 3.4$ min) corresponded with media composition (negative controls, which had only mineral medium), while the second peak (retention time, $\sim 7.4 \mathrm{~min}$ ) corresponded with TNT (when compared with mineral medium containing TNT). The area under the curve of TNT peak was reduced as incubation time advanced, but there was no appearance of any other new peaks at the above stated HPLC set up (Figure 2b). There was a complete disappearance of TNT peak during course of $30 \mathrm{~h}$ of incubation of culture. Figure $2 \mathrm{~b}$ represents an overlay of the result of HPLC analysis of disappearance of TNT at various time points $(0,2,10,18,24,30 \mathrm{~h})$ during $S$. typhimurium cultivation in mineral medium.

\section{Nitrite release from the TNT}

S. typhimurium transformed TNT from mineral medium over $72 \%$ in $12 \mathrm{~h}$, and $>95 \%$ in $18 \mathrm{~h}$, and $100 \%$ in $30 \mathrm{~h}$ (Figure $2 \mathrm{a}$, $2 b)$. Nitrite assays were performed to detect the release of $\mathrm{NO}_{2}$ group from TNT, a positive evidence of TNT demineralization. The results of nitrite assays are presented graphically in Figure 2c. The findings of nitrite release corresponded to disappearance of TNT from culture media with incubation time. The absence of nitrite in $S$. typhimurium cultures with- 
out TNT, and in abiotic controls demonstrated that the presence of nitrite in medium was a result of bacterial transformation of TNT.

\section{DISCUSSION}

Enrichment under nitrogen limiting condition facilitates the selection of bacterial isolates that release nitrite or ammonia by degradation of substrate such as TNT. Salmonella typhimurium, Klebsiella pneumoniae, and Acinetobacter baumannii, all are opportunistic, and grow under variable growth conditions. Surprisingly the isolates which tolerated, and grown in TNT containing medium, utilized TNT without
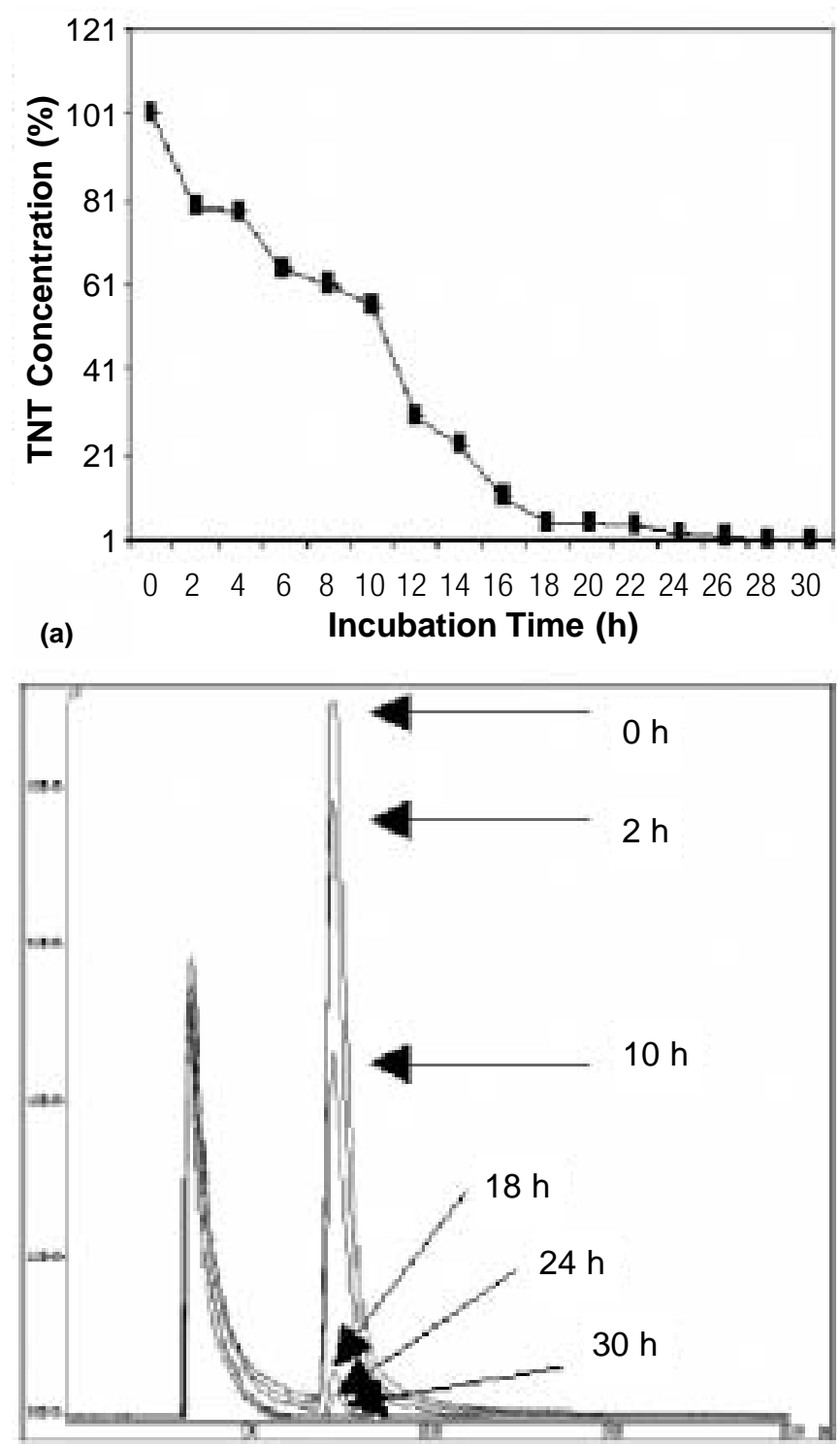

(b) use of any growth stimulant or enhancer, were from clinical specimens. The only clinical isolate of $A$. baumannii showed efficient biotransformation exclusively in the presence of $0.1 \%$ yeast extract. These findings are in agreement with earlier reports. ${ }^{[176]}$ All environmental isolates other than two (which degraded $<5 \mathrm{mg} / \mathrm{l}$ ) that required growth enhancer (data not shown) for TNT transformation failed to transform TNT in either conditions. This study included focus on $S$. typhimurium because of its better ability to remove TNT by consuming it as sole nitrogen source during growth. The growth and viability was unaffected by $20 \mathrm{mg}$ (for $K$. pneumoniae) or $40 \mathrm{mg}$ (for $A$. baumannil) or $50 \mathrm{mg}$ (for $S$. typhimurium) of TNT per liter. The concentrations of TNT beyond these points were inhibitory to isolates. An increase in bacterial growth and protein content is a positive evidence of TNT utilization from enriched medium (Figure 1).

During degradation process of TNT by $S$. typhimurium, a pinkish red to brown color appeared, which gradually darkened around $10 \mathrm{~h}$ of incubation, but disappeared after further incubation. From previous reports of TNT degradation, the hydride-Meisenheimer complex of TNT (H- -TNT) showed a redbrown color by aerobic bacteria, and some of the fungi. ${ }^{[11,18-20]}$ Vorbeck et al. ${ }^{[20]}$ also reported the reduction of TNT to $\mathrm{H}^{-}$TNT, which subsequently gave yellow product by whole cells of Rhodococcus erythropolis and Mycobacterium sp. The yellow products were identified as the protonated and dissociated forms of the $\mathrm{C}-3, \mathrm{C}-5$ dihydride-Meisenheimer complex of TNT (2H-TNT). In our studies both these color changes were noted. Nitroreductase-like activity is not surprising, since the aromatic nitro groups of TNT are extremely susceptible to reduction and can be reduced by a variety of

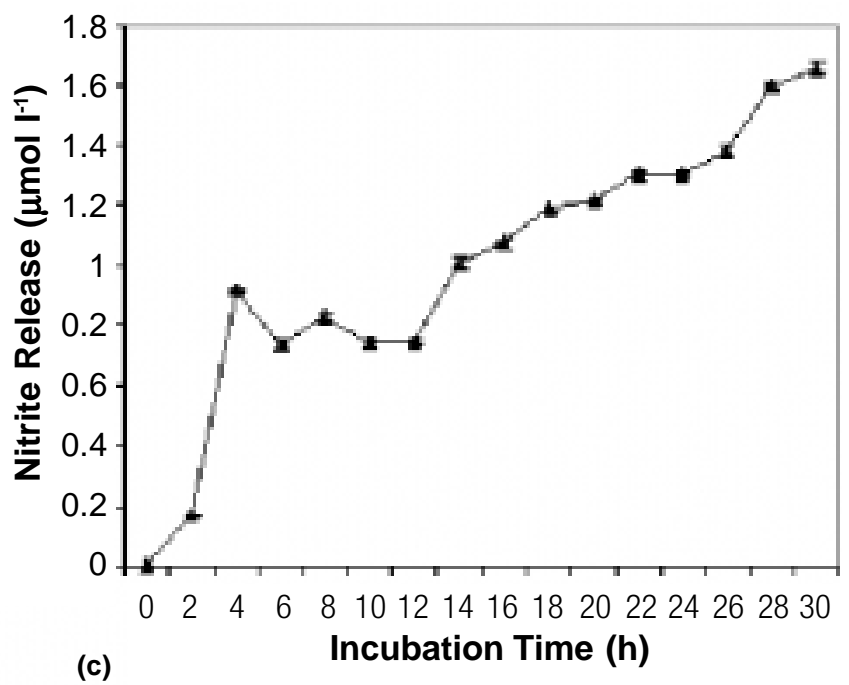

Figure 2: A graphical presentation of evidence, showing utilization of TNT as sole and limiting source of nitrogen during growth of S. typhimurium in rich mineral medium. (a) is graphical presentation of HPLC data, showing a percentage of TNT presented in mineral medium during growth at given time points. (b) is HPLC graphical overlay of crucial time points during growth. A second peak (retention time, $7.4 \mathrm{~min}$ ) corresponds with TNT, while the first with mineral medium (retention time, $3.4 \mathrm{~min}$ ) at Imax $230 \mathrm{~nm}$. (c) is a graph shows release of nitrite during TNT degradation by this strain. There was a high amount of nitrite present in mineral medium during growth. (Error bar is standard error mean) 
oxidoreductases. ${ }^{[5]}$ Nitrite and Dinitrotoluenes (DNTs) are the products of transformation of dihydride-Meisenheimer complex. ${ }^{[19]}$ In our studies we proposed that a $\mathrm{H}^{-}$-TNT like metabolite might have formed as a red-brown colored intermediate products, which probably led to a yellowish $2 \mathrm{H}-\mathrm{TNT}$ -like metabolite, during TNT degradation by $S$. typhimurium, with a concomitant release of nitrite and disappearance of TNT from mineral medium (Figure 2), suggests the involvement of denitration pathway.

The utilization of TNT as sole and limiting source of nitrogen was observed through HPLC data analysis. Figure $2 b$ is an overlay of selected incubation time points' HPLC peaks for TNT (retention time $7.4 \mathrm{~min}$ ), which got reduced as incubation time for culture advanced. By the end of $12 \mathrm{~h}$ about $72 \%$ TNT, and $18 \mathrm{~h}>95 \%$ TNT was transformed, and at $30 \mathrm{~h}$ of incubation almost $100 \%$ TNT was removed from culture medium (Figure 2a). The disappearance of TNT and concurrently appearance of nitrite is highly indicative of denitration. Under these experimental conditions there was no appearance of any new peaks corresponding to intermediate metabolites. DNT was also efficiently degraded by these organisms under similar conditions, is indicative of possible involvement of mineralization pathway during TNT degradation, and one of the intermediates could be DNT. Although there was growth and increasing concentrations of protein contents, the amount nitrite release was increasing over the period of time during incubation, shows that the nitrite release was much higher than proportionate depletion (Figure 2c).

Several researchers have reported TNT degradation by aerobic bacteria, which use denitration pathway ${ }^{[2,4,20,21]}$ and showed that TNT denitration is profitable to bacteria. ${ }^{[22,23]}$ The level of denitration was substantially higher in static cultures than in orbital shaken cultures (data not shown). In fact, orbital agitation of TNT enriched culture was detrimental to TNT degradation, in contrary to $\left(\mathrm{NH}_{4}\right)_{2} \mathrm{SO}_{4}$-supplemented cultures. This showed that static culturing is required for TNT transformation to $\mathrm{H}^{-}-\mathrm{TNT}$ to nitrite in our set up. The formation of red-brown color compound was also not seen during orbital agitation supports this explanation. Although we did not estimate the accumulated compounds of TNT or its intermediate products associated with the whole cells of $S$. typhimurium, a release of high amounts nitrite, and corresponding increased in bacterial growth as well as their protein contents are sufficient to correlate the use of TNT as sole $\mathrm{N}$ source under limiting conditions.

Although the intermediate products of biotransformation have not been defined, an increase in bacterial growth and protein contents, and the release and accumulation of nitrite in mineral medium as TNT is degraded, is a strong indication that this organism has an enzymatic machinery to degrade TNT, and utilize the nitrogen, released out of denitration. In clas- sical pathway most aerobic bacteria follow that degrade TNT, dihydride-Meisenheimer complex $\left(2 \mathrm{H}^{-}-\mathrm{TNT}\right)+$ hydrideMeisenheimer complex $\left(\mathrm{H}^{-}-\mathrm{TNT}\right)+$ protonated $2 \mathrm{H}^{-}-\mathrm{TNT}\left(2 \mathrm{H}^{-}\right.$ $-\mathrm{TNT}^{+} \mathrm{H}^{+}$) are formed, which subsequently undergo $2 \mathrm{e}^{-}$reduction followed by re-aromatization to form DNTs with concomitant release of nitrite. The isomers of DNTs undergo either hydroxylation or dioxygenation to further mineralize into methylcatechol and nitrite release. The methylcatechol is suitable for further ring cleavage by aerobes and utilize as $\mathrm{C}$ and $\mathrm{N}$ source during growth. Vorbeck et al. ${ }^{[20]}$ showed that the ability to utilize TNT diminished with successive subculturing, suggesting sustained use of TNT as N source might not occur. But during our studies we noticed that the TNT degrading ability is sustained for more than 12 successive passages, as well as prolonged storage at $-80^{\circ} \mathrm{C}$ (for 2 year), did not affect this ability. The growth and viability of $S$. typhimurium was also not affected by intermediate metabolites during TNT degradation, suggests that they have been rapidly cleaved further into non-toxic products during growth of this strain. Therefore this strain may prove to have good TNT transforming potential. To best of our knowledge this is the first report on Salmonella species transforming 100\% (of $50 \mathrm{mg} / \mathrm{l}$ ) TNT aerobically, and its possible usefulness in the bioremediation of TNT.

\section{CONCLUSION}

TNT is an environmental toxin, which needs to be removed from contaminated sites to prevent its toxicity on ecosystem. Bioremediation is an effective and eco-friendly method of removal of TNT from natural occupational and environmental sites. Bacterial degradation of TNT by aerobic method is easy to maintain than anaerobic system of cultivation. Salmonella typhimurium, Klebsiella pneumoniae, and Acinetobacter baumannii have not been reported earlier for using TNT as sole source of nitrogen with concomitant release of nitrites and therefore it is logical that these isolates may be having potential for TNT degradation.

\section{ACKNOWLEDGEMENT}

This research work was funded by, the Defence Research and Development Organization (DRDO), Ministry of Defence, India (Grant No. RDR-PX-94/ ERD-1004.02) granted to VSG and SGJ. We thank Institute of Microbial Technology, Chandigarh (Department of Biotechnology, Govt. of India) for help in confirmation of bacterial identification, and Dr. V. N. Krishnamurthy, Director, DRDO-UoP cell, Pune, India, for his cooperation.

\section{REFERENCES}

1. Talmage SS, Opresko DM, Maxwell CJ, et al. Nitroaromatic munitions compounds: Environmental effects and screening values. Rev Environ Contam Toxicol 1999;161:1-156.

2. Kalafut T, Wales ME, Rastogi VK, et al. Biotransformation patterns of 2, 4, 6- trinitrotoluene by aerobic bacteria. Curr Microbiol 1998;36:45-54. 
3. Emery DD, Faessler PC. First production-level bioremediation of explosives-contaminated soil in the United States. Ann N Y Acad Sci 1997;829:326-40.

4. Esteve-Nunez A, Caballero A, Ramos JL. Biological degradation of 2, 4, 6-Trinitrotoluene. Microbiol Mol Biol Rev 2001;65:335-52.

5. French CE, Nicklin S, Bruce N. Aerobic degradation of 2, 4, 6-Trinitrotoluene by Enterobacter cloacae PB2 and by Pentaerythritol Tetranitrate Reductase. Appl Environ Microbiol 1998;64:2864-8.

6. Regan KM, Crawford RL. Characterization of $\mathrm{Cl}$. bifernentans and its biotransformation of TNT and 1,3,5-triazo-1,3,5-trinitrocyclohexane (RDX). Biotechnol Lett 1994;16:1081-6.

7. Binks PR, Nicklin S, Bruce NC. Degradation of hexohydro-1, 3, 5triazine (RDX) by Stenotrophomonas maltophilia PB1. Appl Environ Microbiol 1995;61:1381-422.

8. Fournier D, Halasz A, Spain J, et al. Determination of key metabolites during biodegradation of hexahydro-1, 3, 5-trinitro-1, 3, 5-triazine with Rhodococcus sp., strain DN22. Appl Environ Microbiol 2002;68:166-72.

9. Coleman NV, Nelson DR, Duxbury T. Aerobic biodegradation of hexahydro-1, 3, 5- trinitro-1, 3, 5- triazine (RDX) as a nitrogen source by a Rhodococcus sp. strain DN22. Soil Biol Biochem 1998;30:1159-67.

10. Schackmann A, Muller R. Reduction of nitroaromatic compounds by different Pseudomonas species under aerobic conditions. Appl Microbiol Biotechnol 1991;34:809-13.

11. Vorbeck C, Lenke H, Fischer P, et al. Identification of a hydrideMeisenheimer complex as a metabolite of 2, 4, 6- trinitrotoluene by a Mycobacterium strain. J Bacteriol 1994;176:932-4.

12. Montpas S, Samson J, Langlois E, et al. Degradation of 2, 4, 6- trinitrotoluene by Serratia marcescens. Biotechnol Lett 1997;19:291-4.

13. Halasz A, Groom C, Zhou E, et al. Detection of explosives and their degradation products in soil environment. J Chromatogr A
2002;963:411-8

14. Bergey's Manual of Systematic Bacteriology. Vol. 1, 1984. Krieg NR, Holt JG, editors, Vol. 2, 1986. Sneath PA, Mair NS, Sharpe ME, Holt JG, editors, Vol. 3, 1989. Staley JT, Bryant MP, Pfennig N, Holt JG, editors. Baltimore, MD: Williams and Wilkins.

15. Forbes BA, Sahm DF, Weissfeld AS, editors. Diagnostic Microbiology, Baily and Scotts's. 10 ${ }^{\text {th }}$ Ed. New York: Mosby 1998.

16. Fuller ME, Manning JF Jr. Aerobic Gram-negative and Gram-positive bacteria exhibit differential sensitivity to and transformation of 2, 4, 6-trinitrotoluene (TNT). Cur Microbiol 1997;35:77-83.

17. Boopathy R, Kulpa CF, Manning, et al. J. F. Biotransformation of 2,4,6trinitrotoluene (TNT) by co-metabolism with various co-substrates: A laboratory scale study. Bioresour Technol 1994;47:205-8.

18. Haidour A, Ramos JL. Identification of products resulting from biological reduction of 2, 4, 6-trinitrotoluene, 2, 4-dinitrotoluene, and 2, 6-dinitrotoluene by Pseudomonas sp. Environ Sci Technol 1996;30:2365-70.

19. Kim HY, Song HG. Transformation and mineralization b 2, 4, 6trinitrotoluence by the white rot fungus Irpex lacteus. Appl Microbiol Biotechnol 2003;61:150-6.

20. Vorbeck C, Lenke H, Fischer P, et al. Initial reductive reactions in aerobic microbial metabolism of 2, 4, 6-trinitrotoluene. Appl Environ Microbiol 1998;64:246-52.

21. Hawari J, Beaudet S, Halasz A. Microbial degradation of explosives: biotransformation versus mineralization. Appl Microbiol Biotechnol 2000;54:605-18.

22. Martin JL, Cornfort SD, Shea PJ, et al. Denitration of 2, 4, 6-trinitrotoluene by Pseudomonas savastanoi. Can J Microbiol 1997;43:447-55.

23. Nishino SF, Paoli GC, Spain JC. Aerobic degradation of trinitrotoluene and pathway for bacterial degradation of 2, 6-dinitrotoluene. Appl Environ Microbiol 2000;66:2139- 47. 